\title{
Quantum effects on dynamics of instabilities in Bose-Einstein condensates
}

\author{
V. A. Yurovsky \\ ITAMP, Harvard-Smithsonian Center for Astrophysics, 60 Garden Street, Cambridge MA 02138 \\ Atomic Physics Division, Stop 8423, National Institute of Standards and Technology, Gaithersburg, MD 20889 \\ School of Chemistry, Tel Aviv University, 69978 Tel Aviv, Israel
}

(September 25, 2001)

\begin{abstract}
Dynamics of fluctuations in unstable Bose-Einstein condensates is analyzed by the solution of approximate operator equations. In the case of a condensate with a negative scattering length the present treatment describes a delay of collapse, in agreement with recent experiments. In the case of a collision of two condensate wavepackets it is shown that quantum effects lead to a Bose enhancement of elastic-scattering losses. In both cases the noncondensate atoms are formed as entangled pairs in squeezed states.

03.75.Fi,03.65.Ud,03.70.+k,03.65.Nk
\end{abstract}

\section{INTRODUCTION}

Recent progress in the physics of Bose-Einstein condensation (BEC) of atomic gases (see Refs. [1] 2] and references therein) stimulated a further development of theoretical methods used previously for the description of superfluidity, superconductivity, and collective effects in heavy atoms and nuclei (see Refs. [3 6]). These methods treat condensates as mean fields, while interactions between particles, according to the Bogolubov theory, lead to formation of fluctuations (see Refs. [7] 10]). In the case of a positive scattering length, the condensate (mean field) is stable, while fluctuations can be treated by using a Bogolubov transformation to quasiparticle states.

Recent experiments 11 14 drew attention to the physics of condensates with a negative scattering length. In this case, a free condensate (with a homogeneous mean field) is unstable, collapsing within a finite time [2], while a trapped condensate is stable at the low occupations of the experiment 11, 12]. In the JILA experiments on Rb [13, 14], the effect of Feshbach resonance (see Ref. [15]) was used for changing the sign of the scattering length. This effect allows the formation of a condensate with a positive scattering length, followed by a study of the dynamics of collapse after applying a sudden change of sign of the scattering length. A formal extension of the Bogolubov transformation to the case of negative scattering lengths would lead to complex energies of the quasiparticles, but actually such a transformation does not exist in this case, as was proven by Bogolubov (see Ref. [5]). Nevertheless, the development of fluctuations can substantially affect the dynamics of an unstable condensate (see the theoretical analysis [16] of the JILA experiments 114).
The present paper describes the dynamics of fluctuations by using the parametric approximation employed in Ref. 17] for a model problem of a two-mode atommolecule condensate, and in Ref. [18] for a description of quantum effects on curve crossing. This method is similar to the parametric approximation used in quantum optics (see Ref. [19]), and in the description of interaction of electromagnetic and matter waves in Refs. [20]. It is also closely related to the linearization approximation in quantum soliton theory (see Ref. [21] and references therein) and to the theory of quantum integrals of motion (see Refs. 22] and references therein). The fluctuations are treated here by a linearization of the exact quantum equations of motion for the field operators. In the case of a stable BEC, the resulting linear equations describe the motion of quantum oscillators, corresponding to the Bogolubov quasiparticles. However, in the case of unstable condensates, these equations describe inverted oscillators, leading to a rapid growth of the fluctuations. This growth remains super-exponential as long as the fluctuations do not reach a macroscopic occupation. This dynamics offers a more precise picture compared to the exponential growth obtained from a Liapunov analysis of the classical mean field. Initially, the dynamics can be described as a quantum process of spontaneous decay of the condensate into the fluctuation vacuum (see Refs. [17, 18]), accompanied by the formation of entangled pairs (see also Refs. [23 25]). As in the case of fluctuations in a stable condensate (see Ref. [26]), the pairs are formed in two-mode squeezed states.

The formally calculated energy of a Bogolubov quasiparticle becomes complex also when the energy of the excitation is lower than the energy of a particle in the condensate state. This case can take place if the condensate itself occupies an excited translational state, as realized recently in the NIST experiments on four-wave mixing of atomic condensate waves [27]. The loss of condensate atoms in such a situation was studied in Ref. [28], using the method of complex scattering lengths, without taking into account second-quantized properties of the atomic scattering. The results of the present theory, which take into account these properties, are in agreement with Ref. [28] at short collision times [see Eq. (62) below]. However, at long collision times the effects of Bose enhancement lead to a substantial increase of the losses [see Eq. (63) below]. Notice that the necessity of accounting for the quantum effects was mentioned in Ref. [28] itself. These effects should appear under the condi- 
tions of the NIST experiments [27]. The present method can describe only qualitatively this case of large losses, comparable in magnitude to the initial occupation. This case may be treated by the incorporation of quantum effects into the slowly-varying-envelope approximation developed in Ref. [28,29].

Quantum effects on the loss incurred by collisions of two condensate wavepackets were studied in Ref. [30], using an approach similar to the present one. However, the results of the present work disagree with those of Ref. [30]. The losses quoted in Ref. [30], which have been evaluated only for short collision times, differ also from the results of Ref. 28]. These disagreements are apparently due to some unjustified neglections in Ref. [30] (see discussion in Sec. IIID).

As in the case of fluctuations in a ground-state condensate, the scattered particles are formed here in entangled (squeezed) states. Therefore the processes considered here may open up possibilities for the formation of an entangled gas remained after the decay of a condensate, or after the collision of wavepackets. The entangled gas can have many application (see Ref. [26]).

This paper is organized as follows. Section II considers the dynamics of instabilities in a ground-state condensate with a negative scattering length. The equations of motion for the condensate and the fluctuations are derived in Sec. IIA by using a projection onto a coherent state. The equations for the fluctuations are solved in Sec. II B. In the dependence on the excitation energy, the fluctuations are divided into two classes, of stable and unstable modes, which are respectively associated with regular and inverted oscillators in Sec. IIC. The dynamics and statistical properties of the instabilities are considered in Sec. IID, and Sec. IIE describes the dynamics of condensate depletion.

The collision of two matter waves is studied in Sec. III. General equations for the condensate and the fluctuations are derived in Sec. IIIA. Section IIIB considers elastic losses in collisions of two matter waves. The collisions of rectangular and Gaussian wavepackets are treated in detail in Secs. III G and III D, respectively.

A system of units in which Planck's constant is $\hbar=1$ is used below.

\section{GROUND-STATE CONDENSATE}

\section{A. Separation the condensate and fluctuations}

Consider a homogeneous gas of bosons of mass $m$, in a finite quantization box of volume $\mathcal{V}$, described by the following Hamiltonian in the momentum representation (see Refs. [迎]),

$\hat{H}=\sum_{\mathbf{p}} \hat{\psi}^{\dagger}(\mathbf{p}, t)[\epsilon(\mathbf{p}) \hat{\psi}(\mathbf{p}, t)$

$$
\left.+\frac{1}{2 \mathcal{V}} \sum_{\mathbf{q}, \mathbf{p}^{\prime}} U(q) \hat{\psi}^{\dagger}\left(\mathbf{p}^{\prime}, t\right) \hat{\psi}(\mathbf{p}-\mathbf{q}, t) \hat{\psi}\left(\mathbf{p}^{\prime}+\mathbf{q}, t\right)\right]
$$

Here $\hat{\psi}(\mathbf{p}, t)$ is the annihilation operator for bosons in the momentum $\mathbf{p}$-mode, $\epsilon(\mathbf{p})=\mathbf{p}^{2} /(2 m)$ is the kinetic energy, and $U(q)$ describes the elastic scattering. In the model case of a zero-range contact potential, $U$ is a constant, and can be expressed in terms of the elastic scattering length $a_{a}$ as

$$
U=4 \pi a_{a} / m .
$$

(A modification of the contact-potential model was recently proposed in Ref. 31] which allows to avoid ultraviolet divergences). The Hamiltonian (11) leads to the following equations of motion for the annihilation operators:

$$
\begin{aligned}
& i \dot{\hat{\psi}}(\mathbf{p}, t)=\epsilon(\mathbf{p}) \hat{\psi}(\mathbf{p}, t) \\
& +\frac{1}{\mathcal{V}} \sum_{\mathbf{q}, \mathbf{p}^{\prime}} U(q) \hat{\psi}^{\dagger}\left(\mathbf{p}^{\prime}, t\right) \hat{\psi}(\mathbf{p}-\mathbf{q}, t) \hat{\psi}\left(\mathbf{p}^{\prime}+\mathbf{q}, t\right)
\end{aligned}
$$

Following a general method [7] 10] let us separate the condensate part by a projection onto a coherent state $|\Phi\rangle$ defined so that

$$
\hat{\psi}(\mathbf{p}, t)|\Phi\rangle=\delta_{\mathbf{p} 0} \bar{\Phi}(t)|\Phi\rangle, \quad\langle\Phi \mid \Phi\rangle=1,
$$

where the mean field $\bar{\Phi}(t)=\langle\hat{\psi}(\mathbf{p}, t)\rangle=\langle\Phi|\hat{\psi}(\mathbf{p}, t)| \Phi\rangle$ describes the ground-state condensate occupying the momentum state $\mathbf{p}=0$. The annihilation operator can be written as

$$
\hat{\psi}(\mathbf{p}, t)=[\Phi(t)|\Phi\rangle\langle\Phi|+\hat{\xi}(\mathbf{p}, t)] \exp (-i U(0) N t / \mathcal{V}),
$$

where $\Phi(t)=\bar{\Phi}(t) \exp (i U(0) N t / \mathcal{V})$, the operators $\hat{\xi}(\mathbf{p}, t)$ represent the non-condensate part (fluctuations) having the zero expectation value,

$$
\langle\hat{\xi}(\mathbf{p}, t)\rangle=0,
$$

and

$$
\begin{aligned}
N & =\sum_{\mathbf{p}}\left\langle\hat{\psi}^{\dagger}(\mathbf{p}, t) \hat{\psi}(\mathbf{p}, t)\right\rangle \\
& =|\Phi(t)|^{2}+\sum_{\mathbf{p}}\left\langle\hat{\xi}^{\dagger}(\mathbf{p}, t) \hat{\xi}(\mathbf{p}, t)\right\rangle
\end{aligned}
$$

is the conserved total number of bosons. The exponential factor in Eq. (5) shifts the zero energy to the energy of non-excited condensate.

Substitution of Eq. (5) into Eq. (3), combined with the property (6), produces equations of motion for $\Phi(t)$ and $\hat{\xi}(\mathbf{p}, t)$. Assuming a small occupation of the fluctuations, let us keep in these equations only terms of lowest 
non-vanishing order in $\hat{\xi}(\mathbf{p}, t)$ and $\hat{\xi}^{\dagger}(\mathbf{p}, t)$, obtaining the following equations:

$$
\begin{aligned}
i \dot{\Phi}(t)= & \frac{1}{\mathcal{V}} \sum_{\mathbf{p}} U(p)\langle\hat{\xi}(\mathbf{p}, t) \hat{\xi}(-\mathbf{p}, t)\rangle \Phi^{*}(t) \\
& +\frac{1}{\mathcal{V}} \sum_{\mathbf{p}} U(p)\left\langle\hat{\xi}^{\dagger}(\mathbf{p}, t) \hat{\xi}(\mathbf{p}, t)\right\rangle \Phi(t) \\
i \dot{\hat{\xi}}(\mathbf{p}, t) & =d(p) \hat{\xi}(\mathbf{p}, t)+g(p) \hat{\xi}^{\dagger}(-\mathbf{p}, t),
\end{aligned}
$$

where

$$
d(p)=\epsilon(p)+U(p) n_{0}, \quad g(p)=U(p) \Phi^{2} / \mathcal{V},
$$

and $n_{0}=|\Phi|^{2} / \mathcal{V}$ is the condensate density. This approximate method, that leads to linear operator equations for the fluctuations, is similar to the parametric approximation used in quantum optics (see Ref. [19]). It has been applied to the study of a molecular BEC dissociation in Refs. [17, 18]), and is valid as long as the depletion of the condensate is negligibly small. An applicability criterion for this method is presented in Sec. IIE below [see Eq. (36)].

The present approximation is gapless [see Eq. (13) below] and, therefore, non-conserving (see Ref. [9]), although the average number of the atoms is conserved [see Eq. (7) above].

\section{B. Stable and unstable modes}

Equation (9) combined with the corresponding equation for $\hat{\xi}^{\dagger}(-\mathbf{p}, t)$ provide a set of two linear operator equations (similar to the set considered in Refs. 17, 18]). This set can be solved exactly, using common methods reducing it to an eigenproblem, by assuming the timeindependence of $d(p)$ and $g(p)$. This assumption is corroborated by Eq. (8), which shows that the variation of $\Phi$ is of the second order in $\hat{\xi}(\mathbf{p}, t)$ and therefore can be neglected. Indeed, the validity of the parametric approximation requires a small variation of the condensate density, and the dominant part of the condensate phase is accumulated in the exponential factor in Eq. (5).

As in the case considered in Ref. [17], the form of the solution depends on the ratio of the detuning $d(p)$ to the coupling strength $g(p)$. All momentum modes observing

$$
|g(p)|>d(p), \text { or } \epsilon(p)+2 U(p) n_{0}<0
$$

are unstable, and the corresponding solutions attain the aperiodic form

$$
\begin{aligned}
\hat{\xi}(\mathbf{p}, t)= & {\left[\cosh (\lambda(p) t)-i \frac{d(p)}{\lambda(p)} \sinh (\lambda(p) t)\right] \hat{\xi}(\mathbf{p}, 0) } \\
& -i \frac{g(p)}{\lambda(p)} \sinh (\lambda(p) t) \hat{\xi}^{\dagger}(-\mathbf{p}, 0)
\end{aligned}
$$

where

$$
\begin{aligned}
\lambda(p) & =\sqrt{|g(p)|^{2}-d^{2}(p)} \\
& =\sqrt{\epsilon(p)\left(-2 U(p) n_{0}-\epsilon(p)\right)} .
\end{aligned}
$$

The condition (11) can be obeyed only when $U(p)<0$, i. e., with a negative scattering length, and for excitations with an energy $\epsilon(p)<2|U(p)| n_{0}$. Otherwise, whenever $|g(p)|<d(p)$, the modes are stable and the solutions are oscillatory functions of the form

$$
\begin{aligned}
\hat{\xi}(\mathbf{p}, t)= & {\left[\cos (|\lambda(p)| t)-i \frac{d(p)}{|\lambda(p)|} \sin (|\lambda(p)| t)\right] \hat{\xi}(\mathbf{p}, 0) } \\
& -i \frac{g(p)}{|\lambda(p)|} \sin (|\lambda(p)| t) \hat{\xi}^{\dagger}(-\mathbf{p}, 0) .
\end{aligned}
$$

In a case of intervening mode $(\lambda(p)=0)$ the solution is a linear function of time

$$
\hat{\xi}(\mathbf{p}, t)=[1-i d(p) t] \hat{\xi}(\mathbf{p}, 0)-i g(p) t \hat{\xi}^{\dagger}(-\mathbf{p}, 0) .
$$

This case can take place for the spurious (Goldstowne) mode with a vanishing excitation energy $(\epsilon(p)=0$, see Refs. [6] [32]), or at $\epsilon(p)=2|U(p)| n_{0}$.

\section{Regular and inverted oscillators}

The stable modes can be treated by the Bogolubov transformation. It is therefore instructive to establish a connection between the present and the Bogolubov methods. The equations of motion (9) can be derived from the following Hamiltonian constituent involving two counterpropagating modes,

$$
\begin{aligned}
& \hat{H}(\mathbf{p})=d(p)\left[\hat{\xi}^{\dagger}(\mathbf{p}, t) \hat{\xi}(\mathbf{p}, t)+\hat{\xi}^{\dagger}(-\mathbf{p}, t) \hat{\xi}(-\mathbf{p}, t)\right] \\
& +g^{*}(p) \hat{\xi}(\mathbf{p}, t) \hat{\xi}(-\mathbf{p}, t)+g(p) \hat{\xi}^{\dagger}(\mathbf{p}, t) \hat{\xi}^{\dagger}(-\mathbf{p}, t) .
\end{aligned}
$$

The transformation

$\hat{\xi}_{1,2}(\mathbf{p}, t)=2^{-1 / 2}(\hat{\xi}(\mathbf{p}, t) \pm \hat{\xi}(-\mathbf{p}, t)) \exp \left(-\frac{i}{2} \arg g(p)\right)$

introduces a new pair of field operators, $\hat{\xi}_{1}(\mathbf{p}, t)$ and $\hat{\xi}_{2}(\mathbf{p}, t)$, whose equations of motion are decoupled by separating the Hamiltonian into $\hat{H}(\mathbf{p})=\hat{H}_{1}(\mathbf{p})+\hat{H}_{2}(\mathbf{p})$, where

$$
\hat{H}_{j}=d \hat{\xi}_{j}^{\dagger} \hat{\xi}_{j}-\frac{1}{2}(-1)^{j}|g|\left(\hat{\xi}_{j}^{\dagger} \hat{\xi}_{j}^{\dagger}+\hat{\xi}_{j} \hat{\xi}_{j}\right), \quad j=1,2 .
$$

(Hereafter in this subsection the arguments $\mathbf{p}$ and $t$ are dropped out, for simplicity's sake.) A system described by the Hamiltonian (18) was solved in Ref. [17], and the solutions have a form similar to Eq. (12), or Eq. (14), according to the ratio of $d$ to $g$.

Let us introduce canonically conjugate operators for the coordinates $\hat{Q}_{j}$ and the momenta $\hat{P}_{j}$, such that $\hat{\xi}_{j}=$ $2^{-1 / 2}\left(\hat{Q}_{j}+i \hat{P}_{j}\right)$, writing out the Hamiltonian (18) as 


$$
\hat{H}_{j}=\frac{d+(-1)^{j}|g|}{2} \hat{P}_{j}^{2}+\frac{d-(-1)^{j}|g|}{2} \hat{Q}_{j}^{2}-\frac{d}{2} .
$$

If $|g|<d$, this Hamiltonian describes a harmonic oscillator and can be transformed into the standard form representing free Bogolubov quasiparticles [the total transformation, incorporating (17), being indeed the Bogolubov transformation]. The motion of the harmonic oscillator is periodic, in agreement with the periodic behavior of the solution (14).

A different situation takes place if the condition (11) holds. In this case one of the coefficients (preceding $\hat{P}_{j}^{2}$ or $\hat{Q}_{j}^{2}$ ) is negative, and the Hamiltonian (19) represents an inverted oscillator. It cannot be transformed to a form representing free quasiparticles, and therefore a Bogolubov transformation cannot exist, in agreement with Ref. [5]. The infinite aperiodic motion of the inverted oscillator is exhibited in Eq. (12) and in the instability of the mode.

In a case of intervening mode $(\lambda(p)=0)$ Eq. (13) gives $d(p)=|g(p)|$, one of the coefficients (preceding $\hat{Q}_{j}^{2}$ or $\hat{P}_{j}^{2}$ ) vanishes, and the Hamiltonian (19) represents a motion without restoring forces or an oscillator with infinitely large mass, respectively (see Ref. [6]). Such systems demonstrate linear time-dependence of $\hat{P}_{i}$ or $\hat{Q}_{j}$, respectively, in agreement with the solution (15). The Bogolubov transformation does not exist in this case as well. In the case of the Goldstowne mode $(p=0)$ the Hamiltonian (16) has already the form (18), and the transformation (17) is not necessary. This mode brings only one term $\hat{Q}^{2}(0, t)$ into the total Hamiltonian, in agreement with Ref. 32] (up to the exchange of the coordinate and the momentum).

\section{Dynamics of instabilities}

Consider the dynamics of fluctuations in an unstable BEC, starting from the state of a pure condensate

$$
\left\langle\hat{\xi}^{\dagger}(\mathbf{p}, 0) \hat{\xi}(\mathbf{p}, 0)\right\rangle=\langle\hat{\xi}(\mathbf{p}, 0) \hat{\xi}(-\mathbf{p}, 0)\rangle=0 .
$$

This problem is not only of a speculative interest; it actually describes the recent JILA experiment [14], in which the elastic scattering length was varied very fast from the zero value (of the ideal condensate) to a negative value (of an unstable condensate). Given the initial conditions (20), Eq. (12) leads to the following dynamics for the occupation of the unstable modes,

$$
\left\langle\hat{\xi}^{\dagger}(\mathbf{p}, t) \hat{\xi}(\mathbf{p}, t)\right\rangle=\frac{|g(p)|^{2}}{\lambda^{2}(p)} \sinh ^{2}(\lambda(p) t),
$$

and for the two-particle correlations,

$$
\begin{aligned}
\langle\hat{\xi}(\mathbf{p}, t) \hat{\xi}(-\mathbf{p}, t)\rangle= & -i \frac{g(p)}{\lambda(p)} \cosh (\lambda(p) t) \sinh (\lambda(p) t) \\
& -\frac{d(p) g(p)}{\lambda^{2}(p)} \sinh ^{2}(\lambda(p) t)
\end{aligned}
$$

It is not surprising that this dynamics is similar to the dynamics of spontaneous dissociation of a molecular condensate, studied in Ref. [17], as the non-condensate modes can be described in terms of the fields $\hat{\xi}_{1,2}(\mathbf{p}, t)$ [see Eq. (17)] by the Hamiltonian (18), which is equivalent to the one considered in Ref. [17]. By analogy to the results of Ref. [17], the non-condensate atoms are formed in squeezed states involving atom pairs of opposite momenta. These states are similar to the two-mode squeezed states of fluctuations in a stable BEC studied in Ref. [26]. The states are perfectly squeezed and have zero variance in the relative number of particles with momenta $\mathbf{p}$ and $-\mathbf{p}$, being optimally entangled in the entropic sense. The uncertainty of the generalized coordinates

$$
\hat{X}_{j}(\mathbf{p}, t)=2^{-1 / 2}\left[\hat{\xi}_{j}(\mathbf{p}, t) \exp \left(i \theta_{j}\right)+\hat{\xi}_{j}^{\dagger}(\mathbf{p}, t) \exp \left(-i \theta_{j}\right)\right],
$$

where the operators $\hat{\xi}_{j}(\mathbf{p}, t)$ (with $\left.j=1,2\right)$ are defined by Eq. (17), attains the maximal and minimal values

$$
\begin{aligned}
\left\langle\hat{X}_{j}^{2}(\mathbf{p}, t)\right\rangle_{ \pm} & =\frac{1}{2} \mid\left[\cosh ^{2}(\lambda(p) t)+\frac{d^{2}(p)}{\lambda^{2}(p)} \sinh ^{2}(\lambda(p) t)\right]^{1 / 2} \\
& \pm\left.\frac{g(p)}{\lambda(p)} \sinh (\lambda(p) t)\right|^{2} \underset{\lambda t \gg 1}{\approx}\left\{\begin{array}{l}
\frac{|g(p)|^{2}}{2 \lambda^{2}(p)} e^{2 \lambda t} \\
\frac{\lambda^{2}(p)}{2|g(p)|^{2}} e^{-2 \lambda t}
\end{array}\right.
\end{aligned}
$$

at the two orthogonal values of the respective phase angles $\theta_{j}$,

$$
\theta_{j \pm}= \pm(-1)^{j} \frac{\pi}{4}+\frac{1}{2} \arctan \left[\frac{d(p)}{\lambda(p)} \tanh (\lambda(p) t)\right] .
$$

A common method for studying BEC stability is the Liapunov analysis (see e. g. Ref. [2]). This method produces equations for small perturbations $\zeta(\mathbf{p}, t)$ to the mean field. These equations are equivalent to Eq. (9), in which the operators $\hat{\xi}(\mathbf{p}, t)$ are replaced by $c$-number functions $\zeta(\mathbf{p}, t)$. In the case studied here one obtains for the Liapunov exponents the expression $\lambda$ given by Eq. (13). This type of analysis attributes an exponential gain to the small perturbation, $|\zeta(\mathbf{p}, t)|^{2} \sim \exp (2 \lambda t)$, when the condition (11) is obeyed. This result is in qualitative agreement with the quantum analysis used here, which attributes an even faster (super-exponential) gain to the fluctuations [see Eq. (21)], as

$$
\frac{d}{d t} \ln \left(\left\langle\hat{\xi}^{\dagger}(\mathbf{p}, t) \hat{\xi}(\mathbf{p}, t)\right\rangle\right)=2 \lambda(p) \operatorname{coth} \lambda(p) t>2 \lambda(p) .
$$

The dynamics of the fluctuations has been studied in Ref. 17]. It is shown there that the quantum (squeezed) state always grows faster than a classical (coherent) 
state. Therefore the Liapunov analysis concerning classical fields underestimates the fluctuation growth, becoming correct only when $\lambda t \gg 1$, where the fluctuations are large enough to be considered as classical ones.

A formal application of the Bogolubov transformation at $U<0$ (although incorrect, as had been observed by Bogolubov himself; see Ref. [5) produces quasiparticles with imaginary energies $i \lambda$, which correspond to exponential growth. However, the transformed field operators do not obey the proper Bose commutation rules.

The homogeneous boson gas with a negative scattering length considered above always has unstable modes due to the continuous nature of the excitation spectrum. The situation changes in the case of a trapped gas having a discrete excitation spectrum. Following Ref. [33], consider $\lambda(p)$ as a function of the condensate density $n_{0}$ for a fixed value of $p=p_{m}$ chosen so that $\epsilon\left(p_{m}\right)=\epsilon_{m}$, where $\epsilon_{m}$ is the energy of an excited state of the trapped condensate. At low condensate density $\lambda\left(p_{m}\right)$ is imaginary, corresponding to the well-known stability of a trapped condensate with a negative scattering length at low occupations (see Ref. [2]). At $n_{0}>\frac{1}{2} \epsilon_{m} /\left|U\left(p_{0}\right)\right|$ the value of $\lambda\left(p_{m}\right)$ becomes real, and therefore describes an instability of the condensate (leading to its collapse). Although the present approach does not take into account the inhomogeneity of the condensate and the variation of its size, the dependence of $\lambda\left(p_{m}\right)$ on $n_{0}$ [see Eq. (13)] is in qualitative agreement with the one obtained from the numerical calculations of Ref. 33] for the monopole mode.

\section{E. Dynamics of the condensate}

Let us consider now the dynamics of condensate depletion. In the limit $\mathcal{V} \rightarrow \infty$, Eqs. (21), (22), and (8) lead to the following equation for the condensate density,

$$
\dot{n}_{0}=-\frac{m p_{s} U^{2} n_{0}^{2}}{2 \pi^{2}}\left(I_{\mathrm{unst}}+I_{\mathrm{stab}}\right)
$$

where

$$
\begin{aligned}
& I_{\mathrm{unst}}=\frac{1}{m p_{s}} \int_{0}^{p_{s}} p^{2} \frac{\sinh (2 \lambda(p) t)}{\lambda(p)} d p \\
& I_{\mathrm{stab}}=\frac{1}{m p_{s}} \int_{p_{s}}^{\infty} p^{2} \frac{\sin (2|\lambda(p)| t)}{|\lambda(p)|} d p
\end{aligned}
$$

are the contributions of the unstable $\left(p<p_{s}\right)$ and stable $\left(p>p_{s}\right)$ modes, respectively,

$$
p_{s}=\sqrt{4 m|U| n_{0}}
$$

is the boundary between the stable and unstable modes, and the contact potential (2) is used hereafter in this section. The statistical weight of intervening mode with $\lambda(p)=0$ is negligible compared to the continuums of the stable and unstable modes. Unlike a case of stable condensate, where the unrestricted behavior of the Goldstowne mode requires a special treatment (see Ref. [32), in the present case the effect of linearly-growing intervening modes is negligible compared to the effect of exponentially-growing unstable modes.

Substitution of $p=p_{s} \cos \frac{\theta}{2}$ allows us to express $I_{\text {unst }}$ in terms of the Weber functions $E_{\nu}(z)$ (see Ref. [34]):

$$
\begin{aligned}
I_{\mathrm{unst}} & =\int_{0}^{\pi} \cos \frac{\theta}{2} \sinh (\tau \sin \theta) d \theta \\
& =i \frac{\pi}{2}\left[E_{1 / 2}(i \tau)+E_{-1 / 2}(i \tau)\right],
\end{aligned}
$$

where $\tau=2 t / t_{\mathrm{NL}}$, and $t_{\mathrm{NL}}=\left(|U| n_{0}\right)^{-1}$ is the nonlinear interaction time (see Ref. [29]). Approximate expressions for $I_{\text {unst }}$, holding at small and large values of $t$, have the following respective forms,

$$
\begin{aligned}
& I_{\text {unst }} \underset{\tau \rightarrow 0}{\approx} \frac{\pi}{2} \frac{\tau}{\sqrt{2} \Gamma(7 / 4) \Gamma(5 / 4)} \\
& I_{\text {unst }} \underset{\tau \rightarrow \infty}{\approx} \frac{1}{2} \sqrt{\frac{\pi}{\tau}} e^{\tau}-\frac{3}{16 \tau} .
\end{aligned}
$$

The contribution of the stable modes can be evaluated by substituting $p=p_{s} \cosh \frac{\theta}{2}$, as a result of which

$$
\begin{aligned}
I_{\text {stab }} & =\int_{0}^{\infty} \cosh \frac{\theta}{2} \sin (\tau \sinh \theta) d \theta \\
& =\frac{1}{2} \sqrt{\frac{\pi}{\tau}} e^{\tau}-i \frac{\pi}{2}\left[E_{1 / 2}(i \tau)+E_{-1 / 2}(i \tau)\right]
\end{aligned}
$$

Its limiting values can be approximately expressed as

$$
\begin{aligned}
& I_{\text {stab }} \underset{\tau \rightarrow 0}{\approx} \frac{\pi}{2}\left(\frac{1}{\sqrt{\pi \tau}}+\sqrt{\frac{\tau}{\pi}}-\frac{\tau}{\sqrt{2} \Gamma(7 / 4) \Gamma(5 / 4)}\right) \\
& I_{\text {stab }} \underset{\tau \rightarrow \infty}{\approx} \frac{3}{16 \tau} .
\end{aligned}
$$

Finally, the rate of condensate depletion can be expressed as

$$
\begin{aligned}
\dot{n}_{0} & =-\frac{m p_{s} U^{2} n_{0}^{2}}{4 \pi \sqrt{\pi \tau}} e^{\tau} \\
& =-4 \sqrt{\frac{2 \pi}{m t}} a_{a}^{2} n_{0}^{2} \exp \left(8 \pi\left|a_{a}\right| n_{0} t / m\right),
\end{aligned}
$$

and the loss of condensate density attains the limiting forms 


$$
\begin{aligned}
\Delta n_{0} & \approx 4 n_{0} \sqrt{\left|a_{a}\right|^{3} n_{0} \tau}=8 \sqrt{\frac{2 \pi t}{m}} a_{a}^{2} n_{0}^{2}, \quad t \ll t_{\mathrm{NL}} \\
\Delta n_{0} & \approx 2 e^{\tau} n_{0} \sqrt{\left|a_{a}\right|^{3} n_{0} / \tau} \\
& =\sqrt{\frac{m}{2 \pi t}} a_{a} n_{0} \exp \left(8 \pi\left|a_{a}\right| n_{0} t / m\right), \quad t \gg t_{\mathrm{NL}}
\end{aligned}
$$

at the two distinguishable loss regimes. At the regime of non-stationarity $t \ll t_{\mathrm{NL}}$ the depletion is mostly due to an occupation of the stable modes, which carry much more statistical weight than the unstable modes $\left[I_{\text {stab }} \gg\right.$ $I_{\text {unst }}$, see Eqs. (31) and (33)]. The statistical weight is infinitely large, leading to a singularity in the depletion rate (34), at $t=0$. This singularity is absent in Eq. (35), and can be eliminated by using of an interatomic interaction with a finite range in place of the contact potential (2). At $t>0$ the occupation of the modes with $p>\sqrt{m / t}$ begins to decrease due to their oscillating behavior, leading to decrease of the depletion rate. At the regime of Bose-Enhancement $t \gg t_{\mathrm{NL}}$ the depletion is dominated by the exponentially increasing occupation of the unstable modes $\left[I_{\text {stab }} \ll I_{\text {unst }}\right.$, see Eqs. (31) and (33)]. The approximate expressions (35) are compared in Fig. 11 to results of numerical integration of Eq. (34).

One should be reminded that the parametric approximation, on which all the analysis above rests, is applicable only as long as $\Delta n_{0} \ll n_{0}$. In the case of one excited mode [17, this approximation is in good agreement with numerical results while $\Delta n_{0} / n_{0}<0.2$. In the case of a dilute condensate $\left(\left|a_{a}\right|^{3} n_{0} \ll 1\right.$, see Ref. [2] $)$, the validity range

$$
t / t_{\mathrm{NL}}<\frac{1}{4}\left|\ln \left(\left|a_{a}\right|^{3} n_{0}\right)\right|
$$

includes a region $t>t_{\mathrm{NL}}$ where the exponential Boseenhancement factor can reach a rather large value, of the order of $\left(\left|a_{a}\right|^{3} n_{0}\right)^{-1 / 2}$.

Equation (35a) predicts small depletion at times $t<$ $t_{\mathrm{NL}}$ (with $\Delta n_{0} / n_{0}<4 \sqrt{\left|a_{a}\right|^{3} n_{0}} \ll 1$, given the dilution parameter $\left|a_{a}\right|^{3} n_{0}$ is small). Therefore, the development of instabilities in BEC, including collapse, starts with a delay time $t \sim t_{\mathrm{NL}}$ after turning on the interatomic interactions, although the collapse itself is a nonlinear phenomenon that cannot be treated by the parametric approximation. Under the conditions of the ${ }^{85} \mathrm{Rb}$ experiment 14 (where $n_{0} \approx 10^{12} \mathrm{~cm}^{-3}$ and $a_{a} \approx-20 \mathrm{~nm}$ ), the expected delay is $t_{\mathrm{NL}} \approx 5 \mathrm{~ms}$ (cf. Fig. 1), in agreement with the experimentally observed delay time for the collapse. In this case the parametric approximation is valid as long as $t<3 t_{\mathrm{NL}}$, allowing for values of the exponential Bose-enhancement factor in Eq. (35b) exceeding 100. It should be noted that the condensate dynamics can be sensitive to initial correlations and the spatial inhomogeneity neglected here.

\section{COLLISIONS OF TWO CONDENSATES}

\section{A. Separation of condensates and fluctuations}

The approach developed in Sec. II above can also be applied to a collision of two free counterpropagating BEC waves with momenta $\pm \mathbf{p}_{0}$ (see Ref. 28]). In this case the annihilation operator should be represented as

$$
\begin{aligned}
\hat{\psi}(\mathbf{p}, t)= & {\left[\Phi_{+}(t)\left|\Phi_{+}\right\rangle\left\langle\Phi_{+}\right| \exp \left(-2 i S_{-}(t)\right)\right.} \\
& +\Phi_{-}(t)\left|\Phi_{-}\right\rangle\left\langle\Phi_{-}\right| \exp \left(-2 i S_{+}(t)\right) \\
& \left.+\hat{\xi}(\mathbf{p}, t) \exp \left(-i S_{+}(t)-i S_{-}(t)\right)\right]
\end{aligned}
$$

where

$$
S_{ \pm}=\frac{U\left(2 p_{0}\right)}{2 \mathcal{V}} \int_{0}^{t}\left|\Phi_{ \pm}\left(t^{\prime}\right)\right|^{2} d t^{\prime}+\frac{U(0)}{2 \mathcal{V}} N t+\frac{1}{2} \epsilon\left(p_{0}\right) t
$$

Here $\Phi_{ \pm}(t)$ describe the two condensate fields with momenta $\pm \mathbf{p}_{0}$, respectively, the coherent states $\left|\Phi_{ \pm}\right\rangle$are defined by

$$
\begin{aligned}
& \hat{\psi}(\mathbf{p}, t)\left|\Phi_{ \pm}\right\rangle=\Phi_{ \pm}(t) \exp \left(-2 i S_{\mp}(t)\right) \delta_{ \pm \mathbf{p}_{0} \mathbf{p}}\left|\Phi_{ \pm}\right\rangle \\
& \left\langle\Phi_{ \pm} \mid \Phi_{ \pm}\right\rangle=1
\end{aligned}
$$

and the conserved total number of atoms is

$$
N=\left|\Phi_{+}(t)\right|^{2}+\left|\Phi_{-}(t)\right|^{2}+\sum_{\mathbf{p}}\left\langle\hat{\xi}^{\dagger}(\mathbf{p}, t) \hat{\xi}(\mathbf{p}, t)\right\rangle .
$$

Substitution of Eq. (37) into Eq. (3) gives the following equations of motion for the fluctuations,

$$
i \dot{\hat{\xi}}(\mathbf{p}, t)=d_{2}(p) \hat{\xi}(\mathbf{p}, t)+g_{2}(p) \hat{\xi}^{\dagger}(-\mathbf{p}, t)+D,
$$

where

$$
\begin{aligned}
& d_{2}(p)= \epsilon(p)-\epsilon\left(\mathbf{p}_{0}\right)+\left[U\left(\left|\mathbf{p}-\mathbf{p}_{0}\right|\right)-\frac{1}{2} U\left(2 p_{0}\right)\right] n_{+} \\
&+ {\left[U\left(\left|\mathbf{p}+\mathbf{p}_{0}\right|\right)-\frac{1}{2} U\left(2 p_{0}\right)\right] n_{-} } \\
& g_{2}(p)=\left[U\left(\left|\mathbf{p}-\mathbf{p}_{0}\right|\right)+U\left(\left|\mathbf{p}+\mathbf{p}_{0}\right|\right)\right] \Phi_{+} \Phi_{-} / \mathcal{V},
\end{aligned}
$$

and $n_{ \pm}=\left|\Phi_{ \pm}\right|^{2} / \mathcal{V}$ are the densities of the condensate waves. The term

$$
\begin{aligned}
D= & \left\{\Phi_{-}^{*}(t) \Phi_{+}(t)\left[U\left(2 \mathbf{p}_{0}\right)+U\left(\left|\mathbf{p}-\mathbf{p}_{0}\right|\right)\right] \hat{\xi}\left(\mathbf{p}-2 \mathbf{p}_{0}, t\right)\right. \\
& \left.+\Phi_{+}^{2}(t) U\left(\left|\mathbf{p}-\mathbf{p}_{0}\right|\right) \hat{\xi}^{\dagger}\left(-\mathbf{p}+2 \mathbf{p}_{0}, t\right)\right\} \\
& \times \exp \left\{2 i\left[S_{+}(t)-S_{-}(t)\right]\right\} \\
+ & \left\{\Phi_{+}^{*}(t) \Phi_{-}(t)\left[U\left(2 \mathbf{p}_{0}\right)+U\left(\left|\mathbf{p}+\mathbf{p}_{0}\right|\right)\right] \hat{\xi}\left(\mathbf{p}+2 \mathbf{p}_{0}, t\right)\right. \\
& \left.+\Phi_{-}^{2}(t) U\left(\left|\mathbf{p}+\mathbf{p}_{0}\right|\right) \hat{\xi}^{\dagger}\left(-\mathbf{p}-2 \mathbf{p}_{0}, t\right)\right\} \\
& \times \exp \left\{2 i\left[S_{-}(t)-S_{+}(t)\right]\right\}
\end{aligned}
$$


describes couplings of fluctuations with momenta different by $2 \mathbf{p}_{0}$. Whenever

$$
p_{0}^{2} \gg 4 m U \sqrt{n_{+} n_{-}}
$$

the disparity of the energies of such fluctuations far exceeds the coupling strengths, and the term $D$ can be neglected in Eq. (41). This allows us also to neglect correlations of such fluctuations in the equations of motion for the condensate fields, which then attain the form

$$
\begin{aligned}
i \dot{\Phi}_{ \pm}(t)= & \frac{1}{\mathcal{V}} \sum_{\mathbf{p}} U\left(\left|\mathbf{p}-\mathbf{p}_{0}\right|\right)\langle\hat{\xi}(\mathbf{p}, t) \hat{\xi}(-\mathbf{p}, t)\rangle \Phi_{\mp}^{*}(t) \\
& +\frac{1}{\mathcal{V}} \sum_{\mathbf{p}} U\left(\left|\mathbf{p}-\mathbf{p}_{0}\right|\right)\left\langle\hat{\xi}^{\dagger}(\mathbf{p}, t) \hat{\xi}(\mathbf{p}, t)\right\rangle \Phi_{ \pm}(t)
\end{aligned}
$$

Equation (41) with $D$ neglected has solutions of the form Eqs. (12), (14), or (15), according to the ratio of $\left|g_{2}\right|$ to $d_{2}$, in which $g, d$, and $\lambda$ are respectively replaced by $g_{2}$, $d_{2}$, and

$$
\lambda_{2}(p)=\sqrt{\left|g_{2}(p)\right|^{2}-d_{2}^{2}(p)} .
$$

The condition of instability $d_{2}(p)<\left|g_{2}(p)\right|$ can be obeyed now even for positive values of the scattering length (and hence of $U$ ), as $d_{2}$ contains a negative term $-\epsilon\left(p_{0}\right)$ [see Eq. (42)]. In the case of a contact potential (2) an instability takes place when

$$
\left|\epsilon(p)-\epsilon\left(\mathbf{p}_{0}\right)+\frac{1}{2} U\left(n_{+}+n_{-}\right)\right|<2 U \sqrt{n_{+} n_{-}},
$$

or when

$$
p_{-}<p<p_{+},
$$

where

$$
p_{ \pm}=\left[p_{0}^{2}-m U\left(n_{+}+n_{-}\right) \pm 4 m U \sqrt{n_{+} n_{-}}\right]^{1 / 2} .
$$

Whenever condition (45) is obeyed, the momenta $p_{ \pm}$are given approximately by

$$
p_{ \pm} \approx p_{0}-\frac{m U}{p_{0}} \frac{n_{+}+n_{-}}{2} \pm \frac{2 m U}{p_{0}} \sqrt{n_{+} n_{-}} .
$$

The instability interval can include the region $p>p_{0}$. It may appear that this fact can lead to the absurd conclusion that a ground-state condensate $\left(p_{0}=0\right)$ with a positive scattering length is unstable. However, the analysis above is valid only when the condition (45) is obeyed. Otherwise, the terms neglected in Eqs. (41) and (46) have to be taken into account, leading to stability of the condensate at $p_{0}=0$.

As in the case of a ground-state condensate, the scattered atoms with momenta $\mathbf{p}$ and $-\mathbf{p}$ are correlated, forming optimally entangled and optimally squeezed states [see discussion following Eq. (22)].

\section{B. Elastic-scattering loss in collisions of two matter waves}

Consider now the elastic-scattering loss of atoms from the two colliding counterpropagating waves bounded only by the normalization box. Substitution of Eqs. (21) and (22) (with the replacement of $d, g$, and $\lambda$ by $d_{2}, g_{2}$, and $\lambda_{2}$, respectively) into Eq. (46) leads to the following equations for the two condensate densities,

$$
\dot{n}_{ \pm}=-\frac{m p_{0} U^{2} n_{+} n_{-}}{\pi^{2}}\left(I_{\mathrm{unst}}+I_{+}+I_{-}\right),
$$

where

$$
\begin{aligned}
& I_{\mathrm{unst}}=\frac{1}{m p_{0}} \int_{p_{-}}^{p_{+}} p^{2} \frac{\sinh \left(2 \lambda_{2}(p) t\right)}{\lambda_{2}(p)} d p \\
& I_{-}=\frac{1}{m p_{0}} \int_{0}^{p_{-}} p^{2} \frac{\sin \left(2\left|\lambda_{2}(p)\right| t\right)}{\left|\lambda_{2}(p)\right|} d p \\
& I_{+}=\frac{1}{m p_{0}} \int_{p_{+}}^{\infty} p^{2} \frac{\sin \left(2\left|\lambda_{2}(p)\right| t\right)}{\left|\lambda_{2}(p)\right|} d p
\end{aligned}
$$

represent the contributions of the unstable $\left(I_{\text {unst }}\right)$ and stable $\left(I_{+}+I_{-}\right)$modes, respectively.

The substitution of

$$
p=\left[p_{0}^{2}-m U\left(n_{+}+n_{-}\right)+4 m U \sqrt{n_{+} n_{-}} \cos \theta\right]^{1 / 2}
$$

allows us to express $I_{\text {unst }}$ in terms of the modified Struve function $L_{\nu}(z)$ (see Ref. 34]), as

$$
I_{\mathrm{unst}}=\int_{0}^{\pi} d \theta \frac{p(\theta)}{p_{0}} \sinh (\tau \sin \theta) \approx \pi L_{0}(\tau),
$$

where $\tau=2 t / t_{\mathrm{NL}}$, and here $t_{\mathrm{NL}}=\left(2 U \sqrt{n_{+} n_{-}}\right)^{-1}$. Approximate expressions for $I_{\mathrm{unst}}$ at small and large values of $\tau$ have the respective forms

$$
I_{\text {unst }} \underset{\tau \rightarrow 0}{\approx} 2 \tau, \quad I_{\text {unst }} \underset{\tau \rightarrow \infty}{\approx} \sqrt{\frac{\pi}{2 \tau}} e^{\tau}-\frac{2}{\tau} .
$$

Given the condition $t p_{0}^{2} / m \ll 1$, the substitution of $\left|\lambda_{2}(p)\right| \approx p^{2} /(2 m)$ produces the estimate

$$
I_{+} \approx \frac{1}{p_{0}} \sqrt{\frac{\pi m}{2 t}} .
$$

The integral $I_{-}$can be estimated under the same condition by retaining only the first term in the Taylor series for $\sin \left(2\left|\lambda_{2}(p)\right| t\right)$, giving $I_{-} \approx 2 p_{0}^{2} t /(3 m)$.

Given the opposite condition, $t p_{0}^{2} / m \gg 1$, the contributions of the stable modes $I_{ \pm}$can be evaluated by substituting 


$$
p=\left[p_{0}^{2}-m U\left(n_{+}+n_{-}\right) \pm 4 m U \sqrt{n_{+} n_{-}} \cosh \theta\right]^{1 / 2},
$$

resulting in

$$
I_{ \pm}=\int_{0}^{\theta_{ \pm}} d \theta \frac{p(\theta)}{p_{0}} \sin (\tau \sinh \theta)
$$

where $\theta_{+}=\infty$ and $p\left(\theta_{-}\right)=0$ (with $\theta_{-} \approx$ $\left.\ln \left[p_{0}^{2} /\left(2 m U \sqrt{n_{+} n_{-}}\right)\right]\right)$. The total contribution of the stable modes, given by

$$
I_{+}+I_{-} \approx \pi\left(I_{0}(\tau)-L_{0}(\tau)\right),
$$

where $I_{0}(z)$ is the modified Bessel function (see Ref. [34), can be approximately expressed as

$$
\begin{aligned}
& I_{+}+I_{-} \approx \pi-2 \tau, \quad m / p_{0}^{2} \ll t \ll t_{\mathrm{NL}} \\
& I_{+}+I_{-} \approx 2 / \tau, \quad t \gg t_{\mathrm{NL}} .
\end{aligned}
$$

Combining Eqs. (52), (54), and (58), one obtains the total rate of the condensate loss

$$
\dot{n}_{ \pm} \approx-\frac{m p_{0} U^{2} n_{+} n_{-}}{\pi} I_{0}(\tau)
$$

Unlike the case of ground-state condensate, characterized by a single time-scale $t_{\mathrm{NL}}$, an additional time-scale, $m / p_{0}^{2}$, appears in the present case, leading to appearance of a third regime of the condensate loss. At short times, $t \ll t_{\mathrm{NL}}$, the loss is dominated by the occupation of the stable modes due to their higher statistical weight.

At the regime of non-stationarity $t p_{0}^{2} / m \ll 1$, the wavefunction of the scattered particles does not have enough time to become stationary, and the estimate 56 ) gives the loss rate

$$
\dot{n}_{ \pm} \approx-\frac{m U^{2} n_{+} n_{-}}{\pi} \sqrt{\frac{m}{2 \pi t}} .
$$

The singularity at $t=0$ has the same nature as in the case of the ground-state condensate with a negative scattering length [see discussion following Eq. (35)].

At the regime of stationarity, $m / p_{0}^{2} \ll t \ll t_{\mathrm{NL}}$, the loss rate becomes time independent, and given by

$$
\dot{n}_{ \pm} \approx-16 \pi a_{a}^{2} \frac{p_{0}}{m} n_{+} n_{-},
$$

in full agreement with theory [28].

At the regime of Bose-enhancement, $t \gg t_{\mathrm{NL}}$, the loss is principally determined by the occupation of unstable modes which increase exponentially due to the effect of Bose enhancement. The loss rate can then be approximately expressed as

$$
\dot{n}_{ \pm} \approx-\frac{\left(2 a_{a}\right)^{3 / 2} p_{0}\left(n_{+} n_{-}\right)^{3 / 4}}{\sqrt{m t}} \exp \left(2 t / t_{\mathrm{NL}}\right) .
$$

The approximate expressions (61), (62), and $(63)$ are compared in Fig. 2 2 to results of numerical integration in Eqs. (53).
The contribution of stable modes decreases at long times as $t$ increases [see Eq. (59b)]. In order to explain the decrease, let us consider the asymptote of $I_{+}+I_{-}$ using, in the spirit of scattering theory [35], the substitution

$$
\begin{array}{r}
\lim _{t \rightarrow \infty} \sin \left(2\left|\lambda_{2}(p)\right| t\right) /\left|\lambda_{2}(p)\right|=\pi \delta\left(\lambda_{2}(p)\right) \\
=\pi\left[\frac{\delta\left(p-p_{+}\right)}{\left|\frac{d}{d p}\right| \lambda_{2}\left(p_{+}\right)||}+\frac{\delta\left(p-p_{-}\right)}{\left|\frac{d}{d p}\right| \lambda_{2}\left(p_{-}\right)||}\right] .
\end{array}
$$

This leads to

$$
I_{+}+I_{-} \sim \frac{\pi}{2 m p_{0}}\left[\frac{p_{+}^{2}}{\left|\frac{d}{d p}\right| \lambda_{2}\left(p_{+}\right)||}+\frac{p_{-}^{2}}{\left|\frac{d}{d p}\right| \lambda_{2}\left(p_{-}\right)||}\right] .
$$

In the absence of many-body effects $(U=0)$, one obtains $\left|\lambda_{2}(p)\right|=\left|p^{2}-p_{0}^{2}\right| /(2 m)$, expressing the regular dispersion law for free particles. Also, $p_{ \pm}=p_{0}$, $\left|\frac{d}{d p}\right| \lambda_{2}\left(p_{ \pm}\right)||=p_{0} / m$, and hence $I_{+}+I_{-}=\pi$, which also hold for small $t$ [see Eq. (59a)]. Therefore the scattering process can be described by a stationary scattering theory at any time $t \gg m / p_{0}^{2}$ (see Ref. 35]). Contrarily, in the case of a finite $U$, many-body effects modify the dispersion law, so that $\left|\frac{d}{d p}\right| \lambda_{2}\left(p_{ \pm}\right)|| \rightarrow \infty$, and hence $I_{+}+I_{-} \rightarrow 0$.

\section{Collisions of rectangular wavepackets}

The discussion above is related to the scattering of two condensate waves bounded only by a normalization box. A more realistic yet simple model is that of a halfcollision of rectangular wavepackets. Consider a homogeneous condensate occupying a region of length $b$ along the $x$ axis (leaving the results independent of size and shape in the $y z$-plane). At the moment $t=0$ a Bragg scattering pulse is applied, creating a wavepacket of density $n_{+}$and momentum $2 \mathbf{p}_{0}$ directed along the $x$ axis, while the rest of the atoms, with density $n_{-}$, remains in the $\mathbf{p}=0$ wavepacket. Losses take place only at the overlapping parts of the two wavepackets. The overlapping time $t_{\text {over }}$ varies from 0 to $t_{\text {col }}$, between the leading and trailing edges of the moving wavepacket, where

$$
t_{\mathrm{col}}=\frac{m b}{2 p_{0}}
$$

is the total duration of the collision of the two wavepackets. The average density loss can be expressed as

$$
\left\langle\Delta n_{ \pm}\right\rangle=-\frac{1}{t_{\text {col }}} \int_{0}^{t_{\text {col }}} d t_{\text {over }} \int_{0}^{t_{\text {over }}} d t \dot{n}_{ \pm}(t)
$$

where $\dot{n}_{ \pm}(t)$ is defined by Eq. (60). 
At the regime of non-stationarity, $t_{c o} p_{0}^{2} / m \ll 1$, or $b p_{0} \ll 1$, the loss rate is given by Eq. (61), and

$$
\left\langle\Delta n_{ \pm}\right\rangle \approx \frac{32}{3} \sqrt{\frac{\pi b}{p_{0}}} a_{a}^{2} n_{+} n_{-} .
$$

At the regime of stationarity, $m / p_{0}^{2} \ll t_{\mathrm{col}} \ll t_{\mathrm{NL}}$, the loss rate is determined by Eq. (62), and the loss has a form

$$
\left\langle\Delta n_{ \pm}\right\rangle \approx 4 \pi a_{a}^{2} b n_{+} n_{-} .
$$

The same expression for the loss can also be obtained by using the method of complex scattering length [28].

At the regime of Bose-enhancement, $t_{\mathrm{col}} \gg t_{\mathrm{NL}}$, the loss rate Eq. (63) gives the average density loss

$$
\left\langle\Delta n_{ \pm}\right\rangle \approx \frac{p_{0}^{5 / 2}}{32 \pi^{2} a_{a}^{1 / 2} b^{3 / 2}\left(n_{+} n_{-}\right)^{1 / 4}} \exp \left(2 t_{\mathrm{col}} / t_{\mathrm{NL}}\right)
$$

The approximate expressions (68), (69), and (70) are compared in Fig. 2 to results of numerical integration in Eqs. (53) and (67).

The parametric approximation and, hence all the analysis above, are applicable only as long as $\left\langle\Delta n_{ \pm}\right\rangle \ll$ $\sqrt{n_{+} n_{-}}$. Using Eq. (70), this condition can be rewritten in the form of a limit on the duration of collision consistent with the approximation:

$$
t_{\mathrm{col}}<\frac{1}{2} t_{\mathrm{NL}} \ln \frac{32 \pi^{2} a_{a}^{1 / 2} b^{3 / 2}\left(n_{+} n_{-}\right)^{3 / 4}}{p_{0}^{5 / 2}} .
$$

For example, in the NIST $\mathrm{Na}$ experiment 27] (where $n_{+} \approx n_{-} \approx 10^{14} \mathrm{~cm}^{-3}, a_{a} \approx 3 \mathrm{~nm}, b \approx 20 \mu \mathrm{m}$, and $p_{0} \approx 10^{5} \mathrm{~cm}^{-1}$; see Ref. [29]), the parametric approximation is applicable while $t_{\mathrm{col}}<3 t_{\mathrm{NL}}$, which allows for values of the exponential Bose-enhancement factor in Eq. (70) of more than a 100. This validity range substantially exceeds the range of applicability of the complex scattering-length method, $t_{\mathrm{col}} \ll t_{\mathrm{NL}}$. However, in the four-wave-mixing NIST experiment [27, $t_{\mathrm{col}} \approx 7 t_{\mathrm{NL}}$, which requires taking into account a considerable depletion of the condensates during the collision. This task may be performed by combining the present approach with that of the slowly-varying-envelope approximation [28,29.

\section{Collisions of Gaussian wavepackets}

The consideration of wavepackets of a more realistic shape requires the solution of Eq. (41) for time-dependent $d_{2}$ and $g_{2}$. This solution can be obtained by using a perturbation theory, which is applicable as long as $t \ll t_{\mathrm{NL}}$, and does not describe the effects of Bose enhancement. The evaluation is made simpler in the coordinate representation, in which case the linearized equations of motion for the field operators of the fluctuations $\hat{\tilde{\xi}}(\mathbf{r}, t)$ have the form

$$
\begin{aligned}
& i \dot{\tilde{\tilde{\xi}}}(\mathbf{r}, t)=\frac{\hat{p}^{2}}{2 m} \hat{\tilde{\xi}}(\mathbf{r}, t)+N \int d^{3} r^{\prime} \tilde{U}\left(\mathbf{r}-\mathbf{r}^{\prime}\right)\left[\left|\varphi\left(\mathbf{r}^{\prime}, t\right)\right|^{2} \hat{\tilde{\xi}}(\mathbf{r}, t)\right. \\
& \left.+\varphi^{*}\left(\mathbf{r}^{\prime}, t\right) \varphi(\mathbf{r}, t) \hat{\tilde{\xi}}\left(\mathbf{r}^{\prime}, t\right)+\varphi(\mathbf{r}, t) \varphi\left(\mathbf{r}^{\prime}, t\right) \hat{\tilde{\xi}}^{\dagger}\left(\mathbf{r}^{\prime}, t\right)\right],
\end{aligned}
$$

where $\varphi(\mathbf{r}, t)$ is a unit-normalized wavefunction describing particles in the colliding wavepackets, $N$ is the total number of particles in the wavepackets, and $\tilde{U}(\mathbf{r})$ is the interatomic interaction potential.

The first-order perturbative solution of Eq. (72) can be expressed in the form

$$
\begin{aligned}
\hat{\tilde{\xi}}_{1}(\mathbf{r}, t)=\int d^{3} r^{\prime} \int_{0}^{t} d t^{\prime}\left[A\left(\mathbf{r}, t ; \mathbf{r}^{\prime}, t^{\prime}\right) \hat{\tilde{\xi}}_{0}\left(\mathbf{r}^{\prime}, t^{\prime}\right)\right. \\
\left.+B\left(\mathbf{r}, t ; \mathbf{r}^{\prime}, t^{\prime}\right) \hat{\tilde{\xi}}_{0}^{\dagger}\left(\mathbf{r}^{\prime}, t^{\prime}\right)\right] .
\end{aligned}
$$

Here the zero-order solution

$$
\hat{\tilde{\xi}}_{0}(\mathbf{r}, t)=\sum_{\mathbf{p}} \hat{\xi}(\mathbf{p}, 0) \chi_{\mathbf{p}}(\mathbf{r}, t)
$$

is expressed in terms of free (plane) waves

$$
\chi_{\mathbf{p}}(\mathbf{r}, t)=\mathcal{V}^{-1 / 2} \exp \left(i \mathbf{p r}-i \frac{\mathbf{p}^{2}}{2 m} t\right)
$$

and the annihilation operators $\hat{\xi}(\mathbf{p}, t)$ for the fluctuation in the momentum representation. As the following equations turn out to be independent of the first term in Eq. (73), including the function $A\left(\mathbf{r}, t ; \mathbf{r}^{\prime}, t^{\prime}\right)$, it is not necessary to specify that function. The second term includes the function

$$
\begin{array}{r}
B\left(\mathbf{r}, t ; \mathbf{r}^{\prime}, t^{\prime}\right)=-i N \int d^{3} r^{\prime \prime} \sum_{\mathbf{p}} \chi_{\mathbf{p}}(\mathbf{r}, t) \chi_{\mathbf{p}}^{*}\left(\mathbf{r}^{\prime \prime}, t^{\prime}\right) \\
\times \tilde{U}\left(\mathbf{r}^{\prime \prime}-\mathbf{r}^{\prime}\right) \varphi\left(\mathbf{r}^{\prime \prime}, t^{\prime}\right) \varphi\left(\mathbf{r}^{\prime}, t^{\prime}\right) .
\end{array}
$$

Considering scattering into the vacuum of fluctuations, such that $\left\langle\hat{\tilde{\xi}}_{0}^{\dagger}(\mathbf{r}, t) \hat{\tilde{\xi}}_{0}\left(\mathbf{r}^{\prime}, t^{\prime}\right)\right\rangle=\left\langle\hat{\tilde{\xi}}_{0}(\mathbf{r}, t) \hat{\tilde{\xi}}_{0}\left(\mathbf{r}^{\prime}, t^{\prime}\right)\right\rangle=0$, and therefore

$$
\left\langle\hat{\tilde{\xi}}_{0}(\mathbf{r}, t) \hat{\tilde{\xi}}_{0}^{\dagger}\left(\mathbf{r}^{\prime}, t^{\prime}\right)\right\rangle=\sum_{\mathbf{p}} \chi_{\mathbf{p}}(\mathbf{r}, t) \chi_{\mathbf{p}}^{*}\left(\mathbf{r}^{\prime}, t^{\prime}\right),
$$

one can express the number of the lost atoms in the form

$$
\begin{aligned}
\Delta N= & -\int d^{3} r\left\langle\hat{\tilde{\xi}}_{1}^{\dagger}(\mathbf{r}, \infty) \hat{\tilde{\xi}}_{1}(\mathbf{r}, \infty)\right\rangle \\
= & -\int d^{3} r \int d^{3} r_{1} \int d^{3} r_{2} \int_{0}^{\infty} d t_{1} \int_{0}^{\infty} d t_{2} B^{*}\left(\mathbf{r}, \infty ; \mathbf{r}_{1}, t_{1}\right) \\
& \times B\left(\mathbf{r}, \infty ; \mathbf{r}_{2}, t_{2}\right) \sum_{\mathbf{p}} \chi_{\mathbf{p}}\left(\mathbf{r}_{1}, t_{1}\right) \chi_{\mathbf{p}}^{*}\left(\mathbf{r}_{2}, t_{2}\right) \\
= & 2 N^{2} \operatorname{Im} T_{00}^{(2)}
\end{aligned}
$$


where

$$
\begin{aligned}
T_{00}^{(2)} & =\int_{0}^{\infty} d t^{\prime \prime} \int_{0}^{\infty} d t^{\prime} \int d^{3} r_{1}^{\prime} d^{3} r_{2}^{\prime} d^{3} r_{1}^{\prime \prime} d^{3} r_{2}^{\prime \prime} \varphi^{*}\left(\mathbf{r}_{1}^{\prime \prime}, t^{\prime \prime}\right) \\
& \times \varphi^{*}\left(\mathbf{r}_{2}^{\prime \prime}, t^{\prime \prime}\right) \tilde{U}\left(\mathbf{r}_{1}^{\prime \prime}-\mathbf{r}_{2}^{\prime \prime}\right) G\left(\mathbf{r}_{1}^{\prime \prime}, \mathbf{r}_{2}^{\prime \prime}, t^{\prime \prime} ; \mathbf{r}_{1}^{\prime}, \mathbf{r}_{2}^{\prime}, t^{\prime}\right) \\
& \times \tilde{U}\left(\mathbf{r}_{1}^{\prime}-\mathbf{r}_{2}^{\prime}\right) \varphi\left(\mathbf{r}_{1}^{\prime}, t^{\prime}\right) \varphi\left(\mathbf{r}_{2}^{\prime}, t^{\prime}\right) .
\end{aligned}
$$

Here

$$
\begin{aligned}
& G\left(\mathbf{r}_{1}^{\prime \prime}, \mathbf{r}_{2}^{\prime \prime}, t^{\prime \prime} ; \mathbf{r}_{1}^{\prime}, \mathbf{r}_{2}^{\prime}, t^{\prime}\right)=-i \sum_{\mathbf{p}_{1}, \mathbf{p}_{2}} \chi_{\mathbf{p}_{1}}\left(\mathbf{r}_{1}^{\prime \prime}, t^{\prime \prime}\right) \chi_{\mathbf{p}_{2}}\left(\mathbf{r}_{2}^{\prime \prime}, t^{\prime \prime}\right) \\
& \quad \times \chi_{\mathbf{p}_{1}}^{*}\left(\mathbf{r}_{1}^{\prime}, t^{\prime}\right) \chi_{\mathbf{p}_{2}}^{*}\left(\mathbf{r}_{2}^{\prime}, t^{\prime}\right) \vartheta\left(t^{\prime \prime}-t^{\prime}\right)
\end{aligned}
$$

and $\vartheta(t)$ is the Heavyside step function. As one can see $G\left(\mathbf{r}_{1}^{\prime \prime}, \mathbf{r}_{2}^{\prime \prime}, t^{\prime \prime} ; \mathbf{r}_{1}^{\prime}, \mathbf{r}_{2}^{\prime}, t^{\prime}\right)$ is a time-dependent Green function for two free particles. Therefore $T_{00}^{(2)}$ is nothing else but the second-order term for the diagonal matrix element of the transition amplitude, obtained by time-dependent perturbation theory for a collision of two particles, each in the state $\varphi(\mathbf{r}, t)$. If the size of the wavepacket obeys $b \gg 1 / p_{0}$, the result can be reduced to a form given by the stationary scattering theory (see Ref. [35]), which is proportional to the imaginary part of the elastic scattering length, in agreement with Ref. [28]. Consider two colliding Gaussian wavepackets described by the wavefunction

$$
\begin{aligned}
\varphi(\mathbf{r}, t)= & {\left[F_{+}\left(\mathbf{r}-\frac{\mathbf{p}_{0}}{m} t\right) e^{i \mathbf{p}_{0} \mathbf{r}}+F_{-}\left(\mathbf{r}+\frac{\mathbf{p}_{0}}{m} t\right) e^{-i \mathbf{p}_{0} \mathbf{r}}\right] } \\
& \times \exp \left(-\frac{p_{0}^{2}}{2 m} t\right)
\end{aligned}
$$

where

$$
\begin{aligned}
F_{ \pm}(\mathbf{r})= & \pi^{-3 / 4} \sqrt{N_{ \pm} / N}\left(b_{x} b_{y} b_{z}\right)^{-1 / 2} \\
& \times \exp \left(-\frac{x^{2}}{2 b_{x}^{2}}-\frac{y^{2}}{2 b_{y}^{2}}-\frac{z^{2}}{2 b_{z}^{2}}\right)
\end{aligned}
$$

and $N_{ \pm}$is the number of particles in each wavepacket. Substituting Eq. (81) into Eq. (79) one can obtain

$$
\Delta N=4 \frac{N_{+} N_{-}}{b_{x} b_{y} b_{z}} \frac{p_{0}}{m} a_{a}^{2} t_{\mathrm{col}},
$$

where

$$
t_{\mathrm{col}}=m\left(\frac{p_{0 x}^{2}}{b_{x}^{2}}+\frac{p_{0 y}^{2}}{b_{y}^{2}}+\frac{p_{0 z}^{2}}{b_{z}^{2}}\right)^{-1 / 2}
$$

is the collision duration. Equation (83) coincide with the expression which can be obtained by using of the complex scattering length method [28] and neglecting the depletion of the wavepackets.

The recent preprint 30], posted during preparation of the present paper, treats the loss of atoms from colliding BEC wavepackets by using an approach similar to the one presented here, with solutions of the form of Eq. (12) published previously in Ref. [17]. However, unjustified neglections of substantial terms in the Hamiltonian lead to an expression for $g_{2}$ which is smaller by a factor of four than the one given here by Eq. (43). This neglection should therefore result in an underestimation of the condensate loss.

The above-mentioned work, Ref. [30], considers collision of Gaussian wavepackets to first-order perturbation theory only, which do not describe the effects of Bose enhancement. Finite analytical expressions of the kind presented here were not derived there, and the results of numerical calculations disagree with results of the complex scattering length method [28] even after the correcting for the missing factor four in $g_{2}$ are taken into account. In contrast, the analytical expression Eq. (83) of the present paper, based on the same approximations, is in an agreement with the complex scattering length method.

\section{CONCLUSIONS}

Fluctuations in BEC are treated by using a parametric approximation for the exact quantum equations of motion for the field operators. Depending on the excitation energy, the fluctuations are divided into stable modes, which can be represented as Bogolubov quasiparticles (field oscillators), and unstable modes, which can be represented as inverted oscillators and demonstrate a rapid growth. Initially the dynamics of unstable modes is a quantum process of spontaneous decay into the vacuum of the fluctuations with a super-exponential rate.

This approach is applied to two physical problems. The first one involves the dynamics of a BEC after a fast change of the scattering length from a non-negative to a negative one. In this case, the experimentally observed 14] delay of the condensate collapse is described. The second problem involves the elastic scattering losses on collision of two BEC wavepackets. At short collision times [see Eq. (62)] the losses obtained here are in agreement with those of the method of complex scattering length 28], with specific reference to the collisions of non-confined waves, of rectangular and of Gaussian wavepackets. At longer collision times [see Eq. (63)] quantum effects lead to Bose-enhancement of the elastic scattering losses. The atoms lost from the condensates in both cases form correlated pairs in optimally entangled and optimally squeezed states.

\section{ACKNOWLEDGMENTS}

The author is very grateful to P. S. Julienne and K. Burnett for helpful discussions. Special appreciation is expressed to A. Ben-Reuven for invaluable discussions and the help in preparation of the present paper. This 
work was partially supported by the National Science Foundation through a grant for the Institute for Theoretical Atomic and Molecular Physics at Harvard University and the Smithsonian Astrophysical Observatory.

[1] A.S. Parkins and D.F. Walls, Phys. Rep. 303, 1 (1998).

[2] F. Dalfovo, S. Giorgini, L.P. Pitaevskii, and S. Stringari, Rev. Mod. Phys. 71, 463 (1999).

[3] E. M. Lifshitz and L. P. Pitaevskii, Statistical Physics, Pt. 2 (Pergamon, Oxford, 1980).

[4] A. L. Fetter and J. D. Walecka, Quantum Theory of Many-body Systems (McGraw, San Francisco, 1971).

[5] N. N. Bogolubov and N. N. Bogolubov, Jr. An Introduction to Quantum Statistical Mechanics (Gordon and Breach, Lausanne, 1994).

[6] J.-P. Blaizot and G. Ripka, Quantum Theory of Finite Systems (MIT Press, Cambridge, MA, 1986).

[7] N. P. Proukakis and K. Burnett, J. Res. NIST 101, 475 (1996).

[8] N. P. Proukakis, K. Burnett, and H. T. C. Stoof, Phys. Rev A 57, 1230 (1998).

[9] A. Griffin, Phys. Rev. B 53, 9341 (1996).

[10] R. Walser, J. Williams, J. Cooper, and M. Holland, Phys. Rev. A 59, 3878 (1999).

[11] C.A. Sackett, J.M. Gerton, M. Welling and R.G. Hulet, Phys. Rev. Lett. 82, 876 (1999).

[12] J. M. Gerton, D. Strekalov, I. Prodan, and R. G. Hulet, Nature (London) 408, 692, (2000).

[13] J. L. Roberts et al., Phys. Rev. Lett. 86, 4211 (2001).

[14] E. A. Donley et al., Nature (London) 412, 295 (2001).

[15] E. Timmermans, P. Tommasini,M. Hussein, and A. Kerman, Phys. Rep. 315, 199 (1999).

[16] R.A. Duine and H. T. C. Stoof, Phys. Rev. Lett. 86, 2204 (2001).

[17] A. Vardi, V. A. Yurovsky, and J. R. Anglin, condmat/0105439 Phys. Rev A (in press).

[18] V. A. Yurovsky, A. Ben-Reuven, and P. S. Julienne, condmat/0108372 Phys. Rev. Lett. (submitted).

[19] M. O. Scully and M. S. Zubairy, Quantum Optics (University Press, Cambridge, 1997).

[20] M. G. Moore and P. Meystre, Phys. Rev. A 59, R1754 (1999); C. K. Law and N. P. Bigelow, Phys. Rev A 58, 4791 (1998).

[21] H. A. Haus and C. X. Yu, J. Opt. Soc. Am. B 17, 618 (2000).

[22] V. V. Dodonov and V. I. Man'ko, in Invariants and Evolution of Nonstationary Quantum Systems, Proceedings of Lebedev Physics Institute Vol. 183, edited by M. A. Markov (Nova Science Publishers, Commack, NY, 1989), p. 3; O. V. Man'ko, Phys. Lett. A 228, 29 (1997).

[23] A. Vardi and J. R. Anglin, Phys. Rev. Lett. 86, 568 (2001).

[24] J. R. Anglin and A. Vardi, Phys. Rev A 64, 013605 (2001).

[25] U. V. Poulsen and K. Molmer, Phys. Rev A 63, 023604
(2001).

[26] D. C. Roberts, T. Gasenzer, and K. Burnett, quant$\mathrm{ph} / 0106060$.

[27] L. Deng et al., Nature (London) 398, 218 (1999).

[28] Y. B. Band, M. Trippenbach, J. P. Burke, and P. S. Julienne, Phys. Rev. Lett. 84, 5462 (2000).

[29] M. Trippenbach, Y. B. Band, and P. S. Julienne, Phys. Rev. A 62, 023608 (2000).

[30] R. Bach, M. Trippenbach, and K. Rzazewski, condmat/0107117.

[31] M. Olshanii and L. Pricopenko, cond-mat 0101275.

[32] M. Lewenstein and L. You, Phys. Rev. Lett. 77 3489, (1996).

[33] R. J. Dodd et al, Phys. Rev. A 54, 661 (1996).

[34] H. Bateman and A. Erdely, Higher Transcendental Functions, Vol. 2 (Mc Graw-Hill, New York, 1953).

[35] M. L. Goldberger and K. W. Watson, Collision Theory (Wiley, New York, 1964).

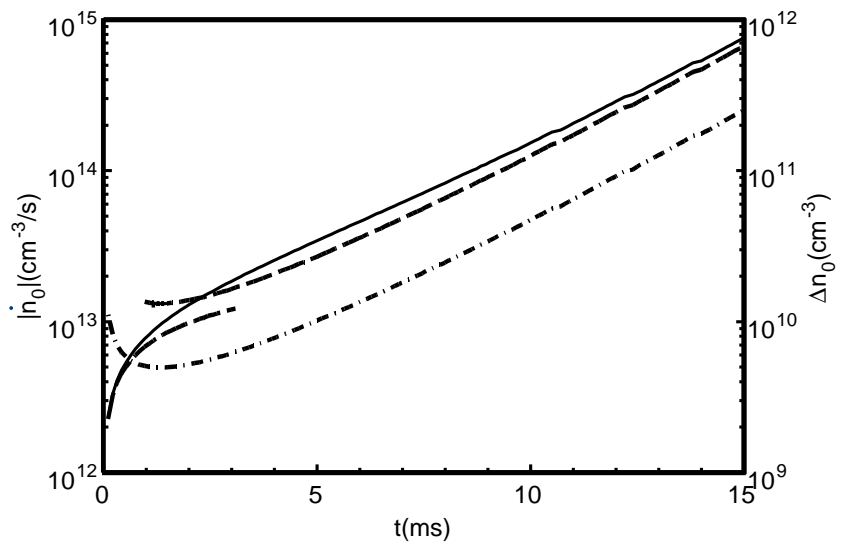

FIG. 1. Time-dependence of the loss of condensate density $\Delta n_{0}$ under conditions of the ${ }^{85} \mathrm{Rb}$ JILA experiment 14 $\left(n_{0} \approx 10^{12} \mathrm{~cm}^{-3}\right)$. The results of numerical integration (solid line) are compared to the approximate equations (35a) and (35b) (dashed line, left and right segments, respectivelly). The dot-dashed line represents the condensate depletion rate $\dot{n}_{0}$ given by Eq. (34). 


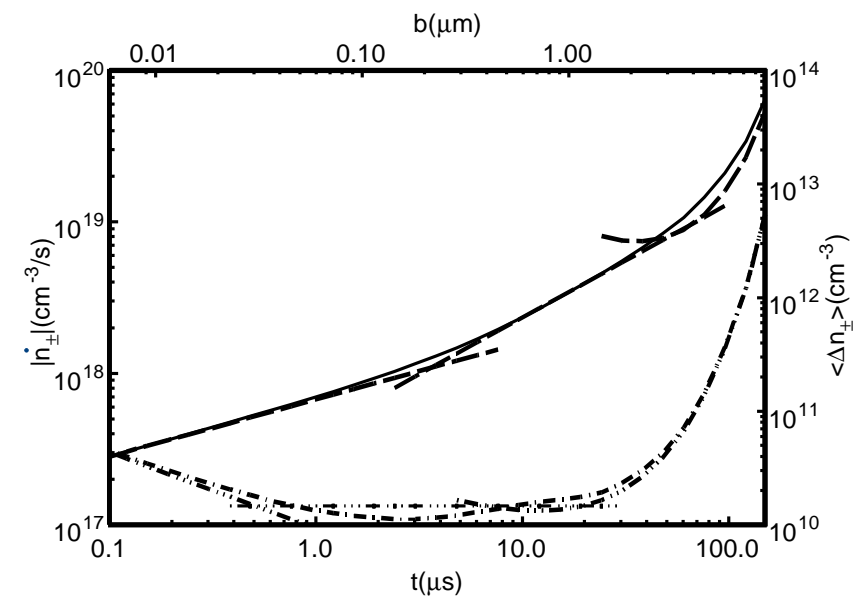

FIG. 2. Time-dependence of the condensate loss rate $\dot{n}_{ \pm}$under conditions of the Na NIST experiment 27 $\left(n_{ \pm} \approx 10^{14} \mathrm{~cm}^{-3}\right)$. The results of numerical integration in Eqs. (53) (dot-dashed line) are compared to the approximate equations (61), (62), and (63) (dot-dot-dot-dashed line, left, central, and right segments, respectivelly). The solid line represents the average density loss $\Delta n_{0}$ for the collision of rectangular wavepackets calculated by using of numerical integration in Eqs. (53) and (67), in comparison with the approximate expressions (68), (69), and (70) (dashed line, left, central, and right segments, respectivelly). 